\title{
Detection of Citrus tristeza virus by using fluorescence resonance energy transfer-based biosensor
}

\begin{abstract}
Due to the low titer or uneven distribution of Citrus tristeza virus (CTV) in field samples, detection of CTV by using conventional detection techniques may be difficult. Therefore, in the present work, the cadmium-telluride quantum dots (QDs) was conjugated with a specific antibody against coat protein (CP) of CTV, and the CP were immobilized on the surface of gold nanoparticles (AuNPs) to develop a specific and sensitive fluorescence resonance energy transfer (FRET)-based nanobiosensor for detecting CTV. The maximum FRET efficiency for the developed nano-biosensor was observed at 60\% in AuNPs-CP/QDs-Ab ratio of $1: 8.5$. The designed system showed higher sensitivity and specificity over enzyme linked immunosorbent assay (ELISA) with a limit of detection of $0.13 \mu \mathrm{gmL}(-1)$ and $93 \%$ and 94\% sensitivity and specificity, respectively. As designed sensor is rapid, sensitive, specific and efficient in detecting CTV, this could be envisioned for diagnostic applications, surveillance and plant certification program
\end{abstract}

Keyword: Bioconjugation; Cadmium-telluride quantum dots; Fluorometric immunoassay; Gold nanoparticles; Nanobiosensor; Quenching 